\title{
Image Steganography for Pixel Prediction using K-nearest Neighbor
}

\author{
Fatima-ezzahra Lagrari \\ Department of Mathematics \\ Ibn Tofail University, \\ Kenitra, Morocco \\ fatimaezzahralagrari3@gmail.com
}

\begin{abstract}
Nowadays to secure the privacy of the patient has increased more research interest during the Image steganography process. Least Significant Bit (LSB) substitute approach was widely exploited to hide the sensitive information in the conventional works. Here, each pixel was reinstated to achieve advanced privacy, other than it increased the complexity. This paper develops a new pixel prediction model-based image steganography to surmount the complication problems widespread in the conventional works. In the proposed pixel prediction model, the K-Nearest Neighbour (KNN) classifier is used to construct the prediction map that recognizes the appropriate pixels for the embedding process. Subsequently, from the medical image to extract the wavelet coefficients based on the Discrete Wavelet Transform (DWT) and embedding power and the undisclosed message is embedded into the HL wavelet band in the embedding phase. At last, from the medical image, the concealed message is extracted by using the DWT. The simulation of the proposed pixel prediction model is carried out by exploiting medical images from the BRATS database. The proposed pixel prediction model has attained maximum performance for the Peak Signal to Noise Ratio (PSNR), Structural Similarity Index (SSIM), and correlation factor, correspondingly.
\end{abstract}

Keywords: Stegnography; Medical Image; Pixel; Secret; Message

\begin{tabular}{ll} 
Nomenclature & \\
\hline Abbreviations & Descriptions \\
\hline PSNR & Peak Signal-To-Noise Ratio \\
CNN & Convolutional Neural Networks \\
BPP & Bits Per Pixel \\
DCT & Discrete Cosine Transform \\
GAN & Generative Adversarial Networks \\
PDASS & Pixeldecimation- Assisted Steganalytic Feature Set \\
ECC & Elliptic Curve Cryptography \\
SEC & Synchronize Embedding- Changes \\
DWT & Discrete Wavelet Transform \\
DNN & Deep Neural Network \\
CBP & Complex Block Prior \\
HPF & High Pass Filter \\
KNN & K-Nearest Neighbor \\
PSO & Particle Swarm Optimization \\
\hline
\end{tabular}

\section{Introduction}

Steganography hides huge numbers of data within a congregation media. For embedment of concealed data, the selected image is called cover or host image, where the ensuing image using embedded concealed data is termed as the stego image [1]. Steganography has three objectives: initially, a huge concealed data is needed; the expression payload indicates concealed data size which might be concealed in a host. The next objective is a confrontation with attacks, and the third objective is the maximum level of protection. Therefore, the best method which used a host image to conceal a secret data ought to be capable to reach 2 significant factors such as maximum stego image visual quality and maximum embedding ability. The competence of some steganographic method can be deliberate by means of 
definite engineering measures, like PSNR and a histogram; these measures estimate the stego image quality. Moreover, the utmost number of secret data bits which might be hidden in an individual pixel of a host image at BPP for the reason of determining the camouflage capability of any suggested method. Nowadays, modern research suggests a novel method for bit substitution to get better camouflage capability of the host image without cooperation stego image quality and to diminish deterioration to the extent that probable [14].

Steganography facilitates us to create communication imperceptible by hiding conceal information in inoffensive cover objects [16]. Image steganography is a secret communication method exploited to broadcast secret messages which encompass embedded into an image. In a digital image to have room for a secret message, the innovative cover image is somewhat enhanced by the embedding approach. Consequently, a stego image is attained. Steganography approaches can be categorized in two concerning the secret message embedding position, such as frequency domain and spatial domain. Nevertheless, it can be additional categorized on cover retrieval nature, image dimension, and on being adaptive based on carrier mediums [15].

Presently, there are two confronts to limit steganography performance. One is the case that additional steganographic information is, not as good as the quality of steganographic images and the lesser the security of the steganographic image. The other is the case that the host image itself is extremely significant, hiding information into noisy and affluent semantic areas are additional safe than information hiding in smooth areas. Hence, to manage with conventional steganography confronts a series of deep models such as CNN [10] and GAN [9] was proposed into a field of image steganography, furthermore broadening field. Taking benefits of this chance, increasingly image steganography based on deep learning was proposed. In [11], a GAN-based steganographic improvement method was proposed that exploits conventional methods to conceal secret messages into the produced image and improve security. Nevertheless, the image produced by this method was semantically deformed and can effortlessly reason suspicion.

This work aspires to develop a new image steganography model by presenting the pixel-based prediction model. Moreover, for the input image, the K-Nearest Neighbor (KNN) is exploited to construct the prediction map. In the proposed image, the stages included a) Steganography system such as a) identification stage, b) embedding stage, and c) extraction stage. In the identification stage, several features, like pixel coverage, wavelet energy, edge information, Gabor feature value, scattering value, and texture component on the basis of the LBP, are obtained from the medical image to calculate the pixels to be embedded. Moreover, the prediction of the pixel model is performed on the KNN classifier that predicts if the recognized pixels are appropriate for the embedding or not. Subsequently, the information of patients is embedded by means of the medical image exploiting the embedding method that uses the DWT coefficient and the embedding strength. At last, from the embedded image the extraction step extracts the undisclosed message using the DWT based extraction method.

\section{Literature Review}

In 2020, Xintao Duan et al [1] proposed a novel high capacity image steganography method on the basis of deep learning. The DCT was exploited to convert the concealed image, and subsequently the transformed image was encrypted through ECC to enhance the anti-detection property of the attained image. To enhance the steganographic capability, SegNet DNN by means of a set of Hiding and Extraction networks enables steganography and extraction of full-size images.

In 2020, Shunquan Tan et al [2], presented the PDASS, a new feature set construction protocol that expands ahead of the new chosen-channel-aware spatial rich method maxSRMd2. This method was based on pixel decimation, a definite kind of image downsampling. On the basis of empirical evaluation and theoretical analysis, the proposed method prejudices synchronization of embedding changes in SEC steganography, and enhances the precision of embedding modify probability estimation was demonstrated.

In 2020, Ayesha Saeed et al [3], proposed a quality-improved and secure method of content-adaptive image steganography. The proposed method was categorized into three chronological orders: pixel complexity, image segmentation, identification, and data embedding. An input cover image is initially separated into small local regions and the pixel-complexity was recognized based on the proposed CBP principle. In a local block, an HPF bank was used and eight remaining responses were attained. After the CBP principle, a complexity level out of nine levels was allocated to an individualized pixel block.

In 2017, A.H. Mohsin1 et al [4], presented a novel method for image steganography on the basis of the PSO method by exploiting pixel selection for concealment of a secret image in the spatial domain, for the reason of high embedment capability. The stego possesses a high level of resistance over a steganalysis attack because of security given by means of image steganography. The purpose of the PSO 
method was to decide an optimal pixel in greyscale host image for concealment of secret bits, as PSO possesses the capability to attain a competent fitness computation.

In 2019, Mustafa Cem Kasapbaşi et al [5], studied the four most important stages. Initially, an example of a Turkish newspaper columnist corpus was collected to attain not only the frequencies of letters and special Turkish characters excluding also punctuations, newlines, spaces, quotations, and so on. Consequently, the initial stage, a static Huffman encoding dictionary was attained for 102 ennumberered characters.

\section{Image Stegnography for Developed Pixel Predicion}

In this section, the proposed pixel prediction model in the medical image to hide the sensitive patient's information is presented. Fig. 1 demonstrates the system model of the proposed pixel prediction based image steganography method.

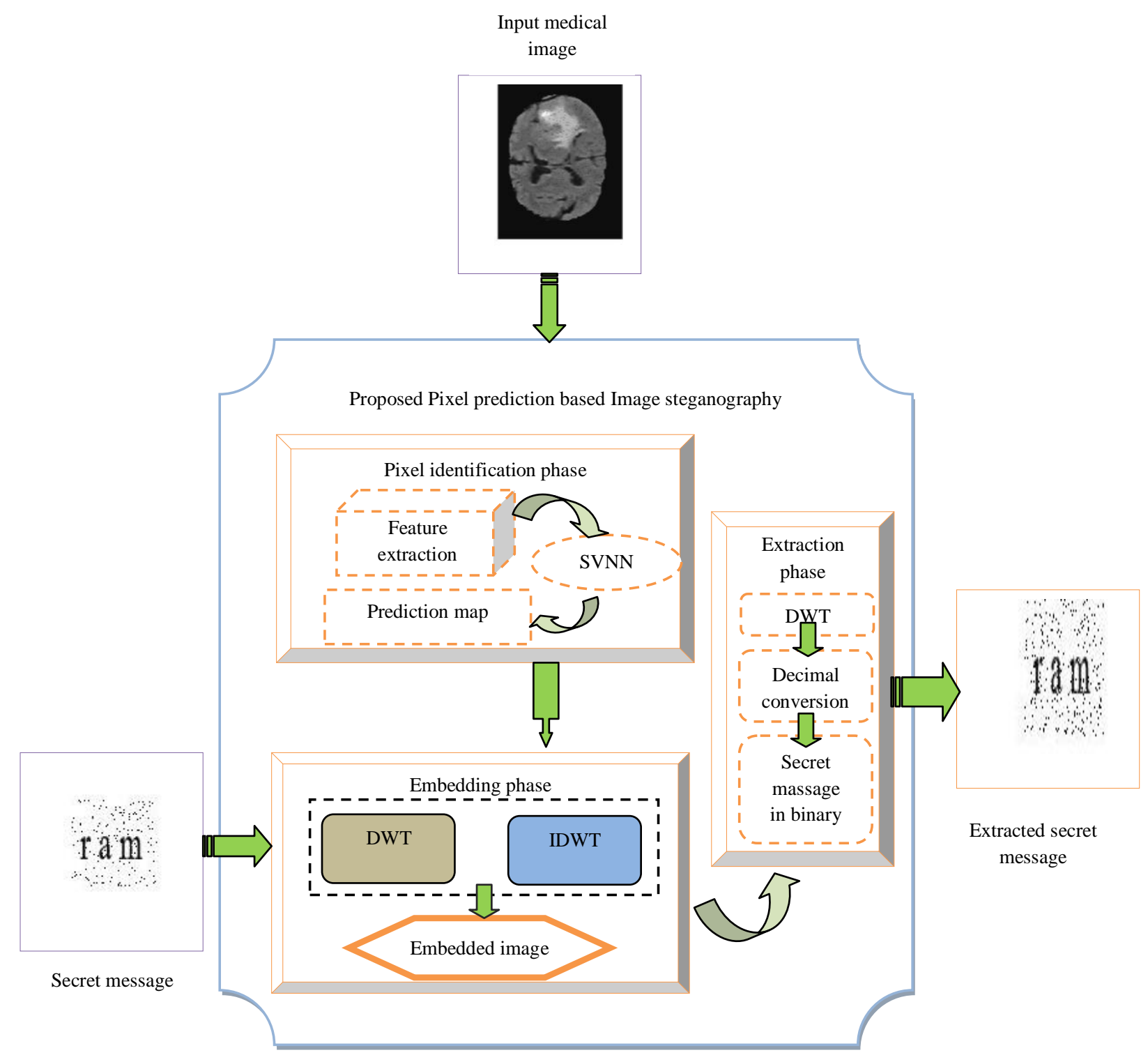

Fig. 1. System model of the proposed pixel prediction based image steganography method.

The proposed pixel prediction on the basis of the image steganography model hides sensitive information in the image during the three following phases, such as the Embedding stage, Identification stage, and the extraction stage. The KNN classifier is included to recognize suitable pixels in input medical image to embed secret information in identification. Several features, like wavelet energy, edge information, pixel coverage, Gabor feature value, scattering value, and texture features are extracted from each pixel of the input image and presented to KNN classifier training. To form the prediction map, the KNN classifier output is gathered, and it comprises the information regarding the appropriate pixels to embed. The prediction map is embedded using the secret image (patient's information) with the 
employ of DWT and the embedding strength in the embedding phase. At last, the secret information obtainable in the embedded image is recognized to find the variation in wavelet coefficients of the embedded and the input image.

\subsection{Identification Stage}

Let the medical image $\mathrm{M}$ of the size $\mathrm{A} \times \mathrm{D}$ is given to the steganography process and the secret information of the patient shown as shas the size of $\mathrm{B} \times \mathrm{G}$. The medical image exploited for the steganography process is an RGB image and therefore, the image pixel values differ from 0 to 255 . In the input image, the secret image to be embedded is indicated in binary form. Mathematical formula of the input image, and the secret image, are shown below,

$$
\begin{gathered}
\mathrm{M}=\left\{\mathrm{m}_{\mathrm{ij}}\right\}, \quad 1 \leq \mathrm{i} \leq \mathrm{A} ; \quad 1 \leq \mathrm{j} \leq \mathrm{D} \\
\mathrm{S}=\left\{\mathrm{s}_{\mathrm{pq}}\right\} ; \quad 1 \leq \mathrm{p} \leq \mathrm{B} ; \quad 1 \leq \mathrm{q} \leq \mathrm{G}
\end{gathered}
$$

whereas, the term $\mathrm{s}_{\mathrm{pq}}$ indicates the pixel in the secret image and $\mathrm{m}_{\mathrm{ij}}$ indicates the pixel in the input image, and it deviates among 0 and 255 , and it has the binary value 0 or 1 .

\subsubsection{Feature extraction}

To identify the appropriate pixels to embed, for the training principle, the KNN classifier is used in this paper; from the input medical image features are extracted. In the medical image $M$, for every pixel 6 features, like wavelet energy $\left(\mathrm{F}_{1}\right)$, pixel coverage $\left(\mathrm{F}_{2}\right)$, edge information $\left(\mathrm{F}_{3}\right)$, scattering value $\left(\mathrm{F}_{4}\right)$, Gabor feature $\left(\mathrm{F}_{5}\right)$, and LBP $\left(\mathrm{F}_{6}\right)$ are extracted. From the pixel, $\mathrm{m}_{\mathrm{ij}}$ the features extracted of the input image is created as the feature vector is shown as

$$
\left[\mathrm{F}_{1}, \ldots, \mathrm{F}_{\mathrm{f}}, \ldots, \mathrm{F}_{6}\right]=\mathrm{F}\left(\mathrm{m}_{\mathrm{ij}}\right)
$$

whereas, F(.)indicates the feature extraction function, and the feature vector, is calculated for the pixel $\mathrm{m}_{\mathrm{ij}}$ of the input image has the size of $[1 \times 6]$. From each pixel the explanation to the diverse features extracted of the input image as follows:

i) Wavelet energy: The input image is the wavelet energy of $M$ is attained by exploiting the DWT [6]. To the input image exploiting the DWT creates four bands, shown as $\left\{\mathrm{N}_{1}, \mathrm{~N}_{2}, \mathrm{~N}_{3}, \mathrm{~N}_{4}\right\}$. Moreover, $\mathrm{N}_{1}, \mathrm{~N}_{2}$, $\mathrm{N}_{3}$ and $\mathrm{N}_{4}$ indicate the LL, LH, HL, and HH bands of the Wavelet transform. Through the DWT the wavelet bands obtained has diverse energy and therefore, wavelet energy of every pixel in the input image $\mathrm{M}$ based upon the band's energy. For the pixel, the wavelet energy feature $\mathrm{m}_{\mathrm{ij}}$ is shown as,

$$
\left\{\mathrm{N}_{1}, \mathrm{~N}_{2}, \mathrm{~N}_{3}, \mathrm{~N}_{4}\right\}=\mathrm{W}\left(\mathrm{m}_{\mathrm{ij}}\right)
$$

whereas, $\mathrm{W}\left(\mathrm{m}_{\mathrm{ij}}\right)$ shows the DWT function. In the proposed work the embedding process is performed focused on the HL band and therefore, the energy utilized by the HL band is deliberated as wavelet energy feature, and it is shown as $\mathrm{F}_{1}=\mathrm{N}_{3}\left(\mathrm{~m}_{\mathrm{ij}}\right)$.

ii) Pixel coverage: It presents the information regarding every pixel coverage value, and therefore, it can be computed as the mean value of the nearby neighbor pixel. Consider pixel $m_{i j} M$ has $L$ number of neighbor pixels in the image, and therefore, pixel coverage of $m_{i j}$ is shown as

$$
\mathrm{F}_{2}=\frac{1}{\mathrm{~L}} \sum_{\mathrm{k}=0}^{\mathrm{L}-1} \mathrm{~m}_{\mathrm{ij}}^{\mathrm{k}}
$$

whereas, $L$ states the neighborhood and $m_{i j}^{k}$ indicates the $k^{\text {th }}$ neighbor pixel of $m_{i j}$.

iii) Edge information: It recognizes if the pixel is an edge/corner pixel or not. This edge information feature obtains the value as 1 if the pixel is an edge pixel; or else it presents the value as 0 . For pixel $\mathrm{m}_{\mathrm{ij}}$ edge information is shown as,

$$
\mathrm{u}(\mathrm{i}, \mathrm{j})=\mathrm{H}\left(\mathrm{m}_{\mathrm{ij}}\right)
$$

whereas, $\mathrm{H}\left(\mathrm{m}_{\mathrm{ij}}\right)$ states the edge information of the pixel $\mathrm{m}_{\mathrm{ij}}$, and it stands for the third feature indicated as $F_{3}=u(i, j)$. It is essential to recognize the edge pixels as the embedding process in the corner pixels might have an effect on the steganography process, and show the way to information loss in the retrieval stage.

iv) Scattering value: From the medical image the scattering coefficients can be attained by using the scattering transform [7]. In the pixel, the scattering transform recognizes the texture information obtainable and it is attained by the convolution of the averaging filter with the pixel. Also, this produces the scattering coefficients of the pixels indicated as, 


$$
J[M]=\|||||\left|M \eta_{\mathrm{v} 1}\right| \otimes \eta_{\mathrm{v} 2}|\cdots| \otimes \eta_{\mathrm{vu}}|\otimes H(\mathrm{u})|
$$

whereas, $\mathrm{H}(\mathrm{u})$ indicates the average filter, and $\eta_{\mathrm{vl}}$ states the filter banks. From the scattering transform of the pixel the features extracted $\mathrm{m}_{\mathrm{ij}}$ are indicated as $\mathrm{F}_{4}=\mathrm{J}\left(\mathrm{m}_{\mathrm{ij}}\right)$.

v) Gabor feature: It can attain by exploiting the Gabor filter [8] to pixels for input medical image. The Gabor feature recognizes the time-frequency position of pixels and presents toughness over diverse contrast and brightness of the image. For the feature extraction generally exploited Gabor filter is 2D Gabor filter, and its filter function, is indicated as

$$
\mathrm{Q}(\mathrm{i}, \mathrm{j}, \theta, \mathrm{w}, \sigma)=\frac{1}{2 \pi \sigma^{2}} \exp \left(-\frac{\mathrm{i}^{2}+\mathrm{j}^{2}}{2 \sigma^{2}}\right) * \exp \left\{2 \pi \mathrm{i}_{\mathrm{m}}(w i \cos \theta+w j \sin \theta)\right\}
$$

whereas, $Q(i, j, \theta, w, \sigma)$ show Gabor filter function for pixel $m_{i j}$ and $i_{m}$ is imaginary part and therefore, have value as $\sqrt{-1}$. In the eq. (8) the term $w$ states the frequency of the sinusoidal wave exploited in the Gaussian filter. The Gabor filter is used with the pixel $\mathrm{m}_{\mathrm{ij}}, \mathrm{Q}\left(\mathrm{m}_{\mathrm{ij}}\right)$ produces the fifth feature $\mathrm{F}_{5}$ of the vector element, and it is indicated as $\mathrm{O}\{\mathrm{i}, \mathrm{j}\}=\mathrm{Q}\left(\mathrm{m}_{\mathrm{ij}}\right)$. Hence, $\mathrm{F}_{5}=\mathrm{O}\{\mathrm{i}, \mathrm{j}\}$.

vi) Textures from LBP: From the image, the LBP [29] features can be attained by using LBP operator with pixels and the ensued features contributes to the sixth feature that is $F_{6}=X(i, j)$. Where, $X(i, j)$ shows texture features attained by LBP operator extraction, and it is indicated as,

$$
X(i, j)=\operatorname{LBP}\left(m_{i j}\right)=\sum_{k=0}^{L-1} 1\left(o_{k}-o_{y}\right) 2^{L}
$$

whereas, $o_{k}$ and $o_{y}$ show the gray value of neighbor and the center pixels, correspondingly, and the function $1(\mathrm{v})$ has the value of 1 or 0 based on subsequent circumstance,

$$
1(\mathrm{v})=\left\{\begin{array}{ll}
1 ; & \mathrm{v} \geq 0 \\
0 ; & \text { else }
\end{array}\right\}
$$

\subsection{Recognition of Pixel Identification: Modelling the Prediction Map with the KNN Classifier}

To find the appropriate pixels to embed is performed by building the prediction map with the KNN classifier. It is a lazy learning method that saves all examples stand for training data points in $\mathrm{n}$ dimensional space. While an unidentified discrete data is received, it verifies the nearest $\mathrm{k}$ number of instances stored (nearest neighbors) and returns the majority widespread class as a prediction for realvalued data it returns mean of KNN.

It weights the contribution of every of $\mathrm{k}$ neighbors consistent with their distance by exploiting better weight to nearest neighbors, in the distance-weighted nearest neighbor algorithm.

$$
\mathrm{w}=\frac{1}{\mathrm{~d}\left(\mathrm{x}_{\mathrm{q}}, \mathrm{x}_{\mathrm{i}}\right)^{2}}
$$

Generally, KNN is vigorous to noisy data as it is averaging the KNN.

\subsubsection{Fitness for the construction of Prediction map}

The KNN uses the minimization function as the fitness, and it focused on selecting the weight values for computing KNN [12] output with the least amount of divergence from the ground value. The phrase for the fitness function of the KNN classifier to build the appropriate prediction map is stated as,

$$
\mathrm{K}=\beta_{\max }+\beta_{\min }+\frac{\mathrm{C}}{\mathrm{A}} \sum_{\mathrm{i}=1}^{\mathrm{A}} \mid \mathrm{Z}_{\mathrm{i}}-\mathrm{Z}_{\mathrm{i}} \text { * }
$$

whereas, C and $\mathrm{Z}_{\mathrm{i}}$ *show the ground regularization factor and the truth information, and the variables $\beta_{\max }$ and $\beta_{\min }$ based upon the Eigenvalue of the weight vectors $\mathrm{T}_{1}$ and $\mathrm{T}_{2}$, and it can be stated as,

whereas, the value of $\beta$ can be stated as,

$$
\begin{gathered}
\beta_{\text {max }}=\max (\beta) \\
\beta_{\text {min }}=\min (\beta)
\end{gathered}
$$

$$
\beta=\text { Eigen }\left(\mathrm{T}^{*} \mathrm{~T}^{\mathrm{T}}\right)
$$

Here, $\mathrm{T}^{\mathrm{T}}$ shows the swap of the weight vector $\mathrm{T}$, and the weight vector encompasses the weight elements in both the input and the hidden layer of KNN. 


\subsection{Training Phase}

In this phase, the Cuckoo Search (CS) is used to train the KNN classifier, to attain the prediction map. In the medical image for each pixel, a total of 6 features are extracted, therefore, to state if the pixel is appropriate for embedding. For the KNN the training process is essential to discover the optimal weights for prediction map construction. CS is the meta-heuristic approach that identifies the optimal solution.

The weights and biases included in the KNN classifier require to be optimally established to characterize characteristics of pixels in the prediction map. Hence, at first, weights, and bias included in the KNN classifier, are arbitrarily produced for the optimization, and it can be stated as,

$$
\mathrm{S}(\mathrm{t})=\left\{\mathrm{S}_{\mathrm{s}}(\mathrm{t}) ; 1 \leq \mathrm{s} \leq \mathrm{e}\right\}
$$

whereas, $S_{s}(t)$ indicates the $s^{\text {th }}$ step size in the CS, and since there are seven weight elements and two bias are included in the KNN classifier, the solution vector size is $\mathrm{e}=9$.

Subsequently, the fitness of every arbitrarily generated solution is analyzed. As fitness is a minimization function, the solution with minimum fitness value is represented to be the superior solution.

\section{(i) Conventional CS approach}

A conventional Cuckoo Search approach can be stated by exploiting the three perfect statements:

a) The cuckoo chooses an arbitrary nest and puts one egg at a time.

b) The optimal nest will progress to the subsequent generations.

c) There are fixed numbers of host nests, and the probability of the host bird recognizing the cuckoo egg is $P_{a}$

$$
\mathrm{P}_{\mathrm{a}} \in[0,1]
$$

To produce a novel solution, $\mathrm{U}^{(\mathrm{t}+1)}$ for cuckoo $\mathrm{i}$, a Levy flight is carried out as below:

$$
\mathrm{U}_{\mathrm{i}}^{\mathrm{t}+1}=\mathrm{U}_{\mathrm{i}}^{\mathrm{t}}+\alpha \oplus \operatorname{levy}(\lambda)
$$

Whereas, $\alpha$ indicates the step size connected by means of the size of the issue $(\alpha>-1) . \lambda$ indicates the scaling parameter, the sign $\oplus$ indicates bit-wise multiplication. Nevertheless, Levy flight is based on the arbitrary walk in that the step size is produced based on the present location and the development option of subsequently states. Levy flight distribution can be used in numerous manners [13]. The Mantegna approach is considered as the approach for symmetric Levy distribution. The step length L indicates the scaling parameter exploited in Mantegna approach is computed as follows:

$$
\mathrm{L}=\frac{\mathrm{p}}{|\mathrm{q}|^{1 / \alpha}}
$$

whereas ${ }^{\mathrm{p}}$ and $\mathrm{q}$ indicate the normally distributed stochastic variables with standard deviations.

$$
\sigma_{\mathrm{p}}=\left[\frac{\Gamma(1+\alpha) \sin (\pi \mathrm{a} \mid 2)}{\Gamma((1+\alpha) / 2) \alpha 2^{(\alpha-1) / 2}}\right] ; \quad \sigma_{\mathrm{q}}=1
$$

The suitable step size must be selected for the appropriate functioning of Levy flights; or else, it will create new solutions that will jump outside the design domain.

\subsection{Testing Phase}

A predicted map is produced by the KNN classifier for the medical test image $\mathrm{M}^{\text {test }}$, based on its feature in the testing phase. From KNN classifier predicted map has two phases 0 and 1 and has a similar size as the input image, and the term for the predicted map is stated as below:

$$
\mathrm{P}=\left\{\mathrm{b}_{\mathrm{ij}}\right\}=\left\{\begin{array}{ll}
1 ; & \text { Pixel suitable for prediction } \\
0 ; & \text { Pixel not suitable for prediction }
\end{array}\right\}
$$

whereas, $b_{i j}$ states the pixels in the prediction map, and it has value 1 if the pixel is appropriate for the prediction otherwise, it encompasses the value as 0 .

\subsection{Embedding Phase}

In the input image, the secret message is embedded, and the predicted map modeled by the KNN classifier acts as a key in the embedding process in the embedding stage. Fig. 2 demonstrates the architecture model of the embedding phase of the proposed pixel-based prediction model. 


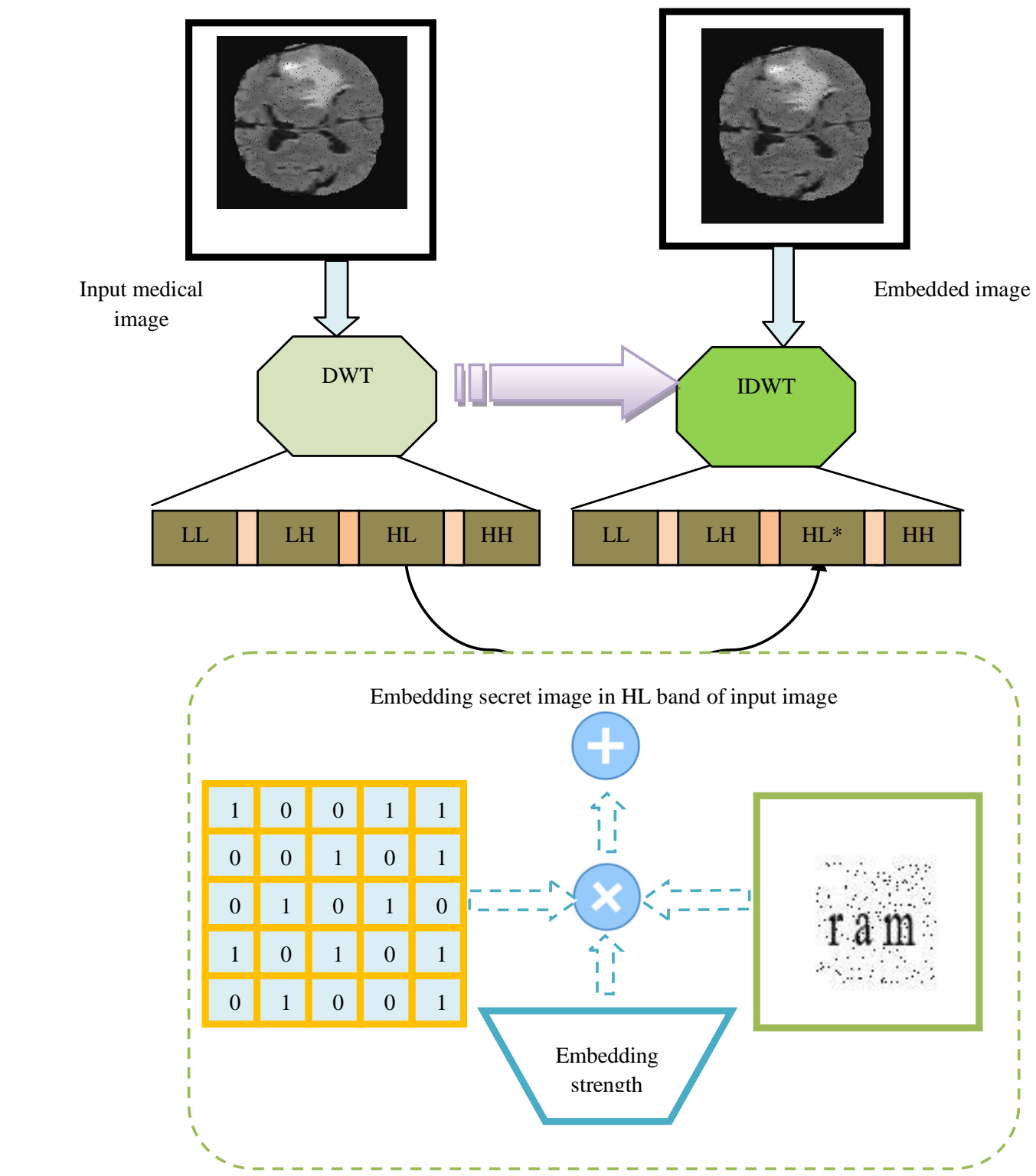

Fig. 2. Architecture model of the embedding phase of the proposed pixel-based prediction model

In the embedding process, the initial step is the DWT application to the input image M. The DWT is additionally beneficial to other methods, like Fourier transform as it recognizes the frequency and position information in the image. To the input image, the DWT transform generates the 4 bands, and it is stated as,

$$
\left[\mathrm{N}_{1}, \mathrm{~N}_{2}, \mathrm{~N}_{3}, \mathrm{~N}_{4}\right]=\mathrm{W}[\mathrm{M}]
$$

where every band takes the wavelet coefficient of the frequency and the energy. The wavelet bands attained via the application of the DWT encompass diverse properties. This paper performs the embedding of the concealed message in the HL band, $\mathrm{N}_{3}$. In $\mathrm{N}_{3}$ the secret message $\mathrm{S}$ is embedded with the employ of the embedding potency and the predicted map from the KNN classifier. From the KNN classifier, the predicted map $\mathrm{P}$ recognizes the appropriate pixels to embed and the embedding power describes the embedding process intensity. The term for embedding a secret message in ${ }^{N_{3}}$ band of the input image is stated as,

$$
\mathrm{N}_{3} *=\mathrm{N}_{3}+\mathrm{S} * \gamma^{*} \mathrm{P}
$$

whereas, $\gamma$ states the embedding potency of the embedding process and $\mathrm{N}_{3} *$ states the band utilizing the secret message embedded. Now, the bands from the DWT after embedding of the secret message into the $\mathrm{N}_{3}$ band is modified as $\left[\mathrm{N}_{1}, \mathrm{~N}_{2}, \mathrm{~N}_{3}{ }^{*}, \mathrm{~N}_{4}\right]$. After embedding a secret message into $\mathrm{N}_{3}$ band, the Inverse DWT (IDWT) is used to the bands. The IDWT to the modified band produces the necessary embedded input image $\mathrm{M}^{*}$ and it is stated as,

$$
\mathrm{M}^{*}=\operatorname{IW}\left[\mathrm{N}_{1}, \mathrm{~N}_{2}, \mathrm{~N}_{3}^{*}, \mathrm{~N}_{4}\right]
$$




\subsection{Extraction Phase}

At last, from the embedded image the secret message is extracted using the DWT to the embedded image $\mathrm{M}^{*}$ in the extraction phase. The embedded image $\mathrm{M} *$ has four wavelet bands beside the secret message in the third band. From the embedded information the secret message can be attained using the DWT to the embedded image, and the process is stated as follows,

$$
\left[\mathrm{N}_{1}^{\mathrm{Er}}, \mathrm{N}_{2}^{\mathrm{Er}}, \mathrm{N}_{3}^{\mathrm{Er} *}, \mathrm{~N}_{4}^{\mathrm{Er}}\right]=\mathrm{W}\left[\mathrm{M}^{*}\right]
$$

whereas, $\mathrm{N}_{1}^{\mathrm{Er}}, \mathrm{N}_{2}^{\mathrm{Er}}$, and $\mathrm{N}_{4}^{\mathrm{Er}}$ show the LL, LH, and HH bands of the DWT process; and $\mathrm{N}_{2}^{\mathrm{Er} *}$ indicates the HL band of the IDWT process from the embedded image during the extraction stage. From the embedded image the secret message can be retrieved to find the difference band attained by DWT of $\mathrm{M}$ and DWT of $\mathrm{M}^{*}$. After the extraction, the secret message attained is stated as,

$$
\mathrm{Y}^{\mathrm{Er}}=\mathrm{N}_{3}^{\mathrm{Er} *}-\mathrm{N}_{3}
$$

From the aforesaid formulation, the secret message attained has a better size than the actual size of the secret message. Therefore, decimal conversion is needed to produce the actual secret message and it is performed using a threshold $\omega$. From $\mathrm{Y}^{\mathrm{Er}}$ the secret message is extracted on the basis of the subsequent circumstance,

$$
\mathrm{S}_{\mathrm{pq}}^{\mathrm{Er}}=\left\{\begin{array}{l}
1 ; \mathrm{Y}^{\mathrm{Er}}>\omega \\
0 ; \text { else }
\end{array}\right\}
$$

whereas, $S_{p q}^{E r}$ states the pixel for the extracted secret message in the binary format. From the extraction phase the secret message is stated as, $S^{\mathrm{Er}}=\left\{S_{\mathrm{pq}}^{\mathrm{Er}}\right\}$, and it has a similar size as the secret message offered for steganography process.

\section{Results and Discussions}

\subsection{Experimental Procedure}

This section summarizes the outcomes of the proposed pixel prediction scheme with the KNN classifier. The experimental outcomes attained by the proposed model for several noises and without noise are shown. The experimentation of the proposed pixel-prediction based image steganography is analyzed with the metrics, such as PSNR, SSIM, and the correlation factor.

\subsection{Performance Analysis}

Table 1 summarizes the comparative evaluation of the proposed prediction model with the KNN classifier and DWT for the image affected by the noise factors. Moreover, the optimal performance of the comparative methods is demonstrated, and from the analysis, it is shown that the proposed prediction model possesses the complete optimal performance. While the image is affected by the noise factors, the proposed prediction model has attained maximum performance for the SSIM, PSNR, and correlation factor, correspondingly.

Table 1: Performance analysis of proposed and conventional models

\begin{tabular}{llll}
\hline Comparative methods & \multicolumn{2}{l}{ Evaluation measures } \\
\cline { 2 - 4 } Random order & SSIM & PSNR (dB) & Correlation factor \\
\cline { 2 - 4 } Sequential order & 0.46 & 8.664 & 0.46 \\
Optimal order & 0.46 & 42.412 & 0.46 \\
CS & 0.46 & 42.222 & 0.46 \\
Proposed & 0.46 & 42.222 & 0.9339 \\
\hline
\end{tabular}

\section{Conclusion}

This paper develops a new pixel prediction based image steganography model using the KNN and the DWT models. The proposed model exploits the medical image to hide the responsive information of the patients. At first, the pixels appropriate to embed the secret message are recognized by training the KNN classifier by means of the features extracted from the medical image. For input image, KNN created the 
prediction map, and by using the DWT coefficients secret message is embedded into input image. Furthermore, for the embedding process, the embedding strength is deliberated. At last, $\mathrm{t}$ from the embedded image he secret message and input medical image are extracted by discovering the dissimilarity in the DWT coefficients of original and the embedded images. The experimentation of the proposed pixel prediction model was examined in several noise circumstances, and the images for the investigation were used from the BRATS database. The proposed pixel prediction model has attained maximum performance for the SSIM, PSNR, and correlation factor, correspondingly.

\section{References}

[1] X. Duan, D. Guo, N. Liu, B. Li, M. Gou and C. Qin, "A New High Capacity Image Steganography Method Combined With Image Elliptic Curve Cryptography and Deep Neural Network," in IEEE Access, vol. 8, pp. 25777-25788, 2020.

[2] S. Tan, H. Zhang, B. Li and J. Huang, "Pixel-Decimation-Assisted Steganalysis of Synchronize-EmbeddingChanges Steganography," in IEEE Transactions on Information Forensics and Security, vol. 12, no. 7, pp. 1658 1670, July 2017.

[3] A. Saeed et al., "An Accurate Texture Complexity Estimation for Quality-Enhanced and Secure Image Steganography," in IEEE Access, vol. 8, pp. 21613-21630, 2020.

[4] A. H. Mohsin et al., "New Method of Image Steganography Based on Particle Swarm Optimization Algorithm in Spatial Domain for High Embedding Capacity," in IEEE Access, vol. 7, pp. 168994-169010, 2019

[5] M. C. Kasapbaşi, "A New Chaotic Image Steganography Technique Based on Huffman Compression of Turkish Texts and Fractal Encryption With Post-Quantum Security," in IEEE Access, vol. 7, pp. 148495-148510, 2019.

[6] Pandey, Prateekshit, Richa Singh, and Mayank Vatsa, "Face recognition using scattering wavelet under Illicit Drug Abuse variations," In Proceedings of International Conference on Biometrics (ICB), Halmstad, pp. 1-6, 2016.

[7] Nikolov, S., Hill, P., Bull, D. and Canagarajah, N., "Wavelets for image fusion," In Wavelets in signal and image analysis, Springer Netherlands, pp. 213-241, 2001.

[8] Kong, W.K., Zhang, D. and Li, W., "Palmprint feature extraction using 2-D Gabor filters," Pattern recognition, vol. 36, no. 10, pp. 2339-2347, 2003.

[9] I. J. Goodfellow, J. Pouget-Abadie, M. Mirza, B. Xu, D. Warde-Farley, S. Ozair, A. Courville, and Y. Bengio, "Generative adversarial net- works," 2014.

[10] Y. Lecun, L. Bottou, Y. Bengio, and P. Haffner, "Gradient-based learn-ing applied to document recognition," Proc. IEEE, vol. 86, no. 11,pp. 2278 2324, Nov. 1998.

[11] D. Volkhonskiy, I. Nazarov, B. Borisenko, and E. Burnaev, "Stegano-graphic generative adversarial networks," 2017.

[12] Jing Chai, Hongwei Liu and Zheng Bao, "A W-KNN classifier to improve radar outlier rejection performance," 2009 IET International Radar Conference, Guilin, 2009, pp. 1-4.

[13] H. R. Soneji and R. C. Sanghvi, Towards the improvement of cuckoo search algorithm, Int. J. Comput. Inform. Syst. Indust. Manage. Appl. 6 (2014), 77-88.

[14] Remmiya R and Abisha C,"Artifacts Removal in EEG Signal Using a NARX Model Based CS Learning Algorithm",Multimedia Research,Volume 1, Issue 1, October 2018.

[15] Vinolin V and Vinusha S,"Edge-based Image Steganography using Edge Least Significant Bit (ELSB) Technique",Multimedia Research,Volume 1, Issue 1, October 2018.

[16] Ninu preetha and Praveena S,"Multiple Feature Sets and SVM Classifier for the Detection of Diabetic Retinopathy Using Retinal Images",Multimedia Research,Volume 1, Issue 1, October 2018. 